Tohoku J. exp. Med., 1983, 140, 301-310

\title{
Immunoelectron Microscopic Examination of IgA Deposition in Dermatitis Herpetiformis
}

\author{
Shin'ichi Masu, Yasuo Tanita, Minoru Igarashi and \\ Makoto SeiJi* \\ Department of Dermatology, Tohoku University School of \\ Medicine, Sendai 980
}

\begin{abstract}
Masu, S., Tanita, Y., Igarashi, M. and SeiJi, M. Immunoelectron Microscopic Examination of $\operatorname{IgA}$ Deposition in Dermatitis Herpetiformis. Tohoku, J. exp. Med., 1983, 140 (3), 301-310 — Ultrastructural localization of IgA in the skin of three Japanese patients with dermatitis herpetiformis (DH) was studied with the immunoelectron microscopic technique using periodate-lysine-paraformaldehyde fixation. In direct immunofluorescence studies, two of the three cases showed fine fibrillar deposition of $\operatorname{IgA}$ and the other case fine granules in the dermal papillae. In the former, the reaction products of $\operatorname{IgA}$ were present in the upper dermis forming various-sized aggregates which were occasionally arranged perpendicularly to the epidermis and appeared to be associated with microfibrillar bundles of the elastic tissue. Reaction products were also deposited to a lesser extent on the microfibrillar component of the elastic fibers at the lower part of the dermal papillae. However, in the latter, the reaction products were found to form smaller aggregates on and around the collagen fibrils rather than on the elastic tissue. Such a localization of IgA reaction products has not yet been reported in DH. The difference of the distribution patterns of $\operatorname{IgA}$ and the possible singularity of Japanese DH cases are discussed _._.... immunoelectron microscopy; IgA; dermatitis herpetiformis
\end{abstract}

Dermatitis herpetiformis (DH), one of the uncommon blistering disorders, has some clinical, histopathological and immunohistopathological characteristics. Among them, IgA deposition in the skin is now thought to be the most reliable factor indicating a diagnosis of DH. So far, two different patterns of IgA deposition have been described in immunofluorescence (IF) studies: 1) the papillary pattern in the dermal papillae and 2) the linear or continuous pattern along the basement membrane (Seah and Fry 1975). The papillary pattern has been also described as "granular pattern" or "fibrillar pattern" according to its appearance. Seah and Fry (1975), however, reported that the difference in appearance is simply due to whether the reticular fibers where $\operatorname{IgA}$ is deposited are sectioned longitudinally or transversely. In fact, the term "granular pattern" has not been fully distinguished from the term "fibrillar pattern" even in immunoelectron microscopic (IEM) studies, and in IEM studies of DH cases with granular or fibrillar IgA deposition, all investigators have reported the reaction products to be associated with microfibrillar bundles and microfibrillar components of the elastic tissue

Received for publication, November 10, 1982.

$*$ Deceased. 
although some differences have been noted (Yaoita and Katz 1976; Stingl et al. 1976; Schmitt et al. 1977; Yaoita 1978; Pehamberger et al. 1979). According to Igarashi et al. (1982), who have reported on Japanese DH cases with the fibrillar IF pattern of IgA deposition, the pattern remained "fibrillar" in appearance even after the tissue specimens were cut at various angles. The same result was obtained for the present three Japanese cases of $\mathrm{DH}$; two of them had the fibrillar and the other fine granular pattern (Masu et al. 1982). The IEM technique was used to ascertain whether the difference in the fluorescence pattern is essential or not.

\section{Materials and Methods}

Cases

Three Japanese patients with DH, a 64-year-old male (Case 1), a 38-year-old female (Case 2) and a 19-year-old female (Case 3), were subjected to study. In each case, pruritic papules and vesicles were distributed on extensor surfaces of the body, occasionally on annular erythema. Erythema and vesicles were induced by KI patch test $(10-50 \%)$ in all cases. Cases 1 and 2 were treated with dapsone with good response. Case 3 was treated with only topical steroid since the eruptions were relatively localized, but papules and vesicles repeatedly recurred. There were no clinical symptoms indicating gluten-sensitive enteropathy in any of the cases, but the absence or presence of this disorder was not ascertained with biopsies of the small intestine. HLA-B8, which has frequently been detected in DH cases in the West (Ery 1979), was not detected in any of the cases.

\section{Tissue specimens}

Biopsy specimens were obtained from the lesional and normal appearing skin of the three cases under local anesthesia. In Cases 1 and 3 , skin biopsies were repeated at about one year after the first biopsies.

\section{Methods}

The specimens were cut into four pieces and were processed according to the following procedures: (1) One piece was fixed in neutral formaldehyde solution and embedded in paraffin for routine histopathological study. (2) The second piece was fixed in $2.5 \%$ glutaraldehyde solution, post-fixed in $2 \%$ osmium tetroxide and processed according to the ordinary electron microscopic procedure. (3) The third piece was quickly frozen in a deep freezer (at $-80^{\circ} \mathrm{C}$ ) and $6 \mu \mathrm{m}$ sections were prepared with a cryostat for direct IF study. (4) The fourth piece was fixed in periodate-lysine-paraformaldehyde (PLP) solution (McLean and Nakane 1974) at $4^{\circ} \mathrm{C}$ for $3 \mathrm{hr}$ for IEM study. The concentration of paraformaldehyde was adjusted to $2 \%$ in PLP solution.

The IF study was carried out with the ordinary IF technique. Fluorescein-conjugated rabbit anti-human IgG, IgA, IgM and $\mathrm{C}_{3}$ (Wehring Institute) were used at dilutions of $1: 20$ or 1:40. Indirect IF study employing anti-IgG and anti-IgA conjugates was also performed on cryostat sections of normal human skin to detect circulating antibodies.

For IEM study, the procedure of McLean and Nakane (1974) was employed. Subsequently to fixation, the tissue strips $(1 \times 1 \times 2 \mathrm{~mm})$ were washed in $0.05 \mathrm{M}$ phosphate buffer $\mathrm{pH} 7.2(\mathrm{~PB})$ containing $7 \%$ sucrose at $4^{\circ} \mathrm{C}$ overnight. Then they were put in $\mathrm{PB}$ containing $15 \%$ sucrose at $4^{\circ} \mathrm{C}$ for $4 \mathrm{hr}$ and in $\mathrm{PB}$ containing $25 \%$ sucrose and $10 \%$ glycerol for $2 \mathrm{hr}$. The tissues were then embedded in an aluminium boat filled with the medium (O.C.T. compound; Miles Laboratories) for frozen tissue specimens and quickly frozen in a mixture of acetone and solid carbon dioxide. Frozen sections $(6 \mu \mathrm{m})$ were cut with a cryostat and placed on egg albumin-coated slides. The slides were dried for about $15 \mathrm{~min}$ at room temperature and exposed to peroxidase-labeled rabbit anti-human IgA antibody 
(DAKO PAP, Lot No. 030) at room temperature for $30 \mathrm{~min}$. The antibody was used at a dilution of 1:20. After being washed in $0.05 \mathrm{M}$ phosphate buffered saline pH 7.2 (PBS) for $20 \mathrm{~min}$, they were immersed into 3-3'diaminobenzidine tetrahydrochloride (Sigma Chemical Company) substrate solution not containing hydrogen peroxide. They were then incubated for $5 \mathrm{~min}$ with the same medium containing $0.01 \%$ hydrogen peroxide (Graham and Karnovsky 1966) and washed in PBS for $20 \mathrm{~min}$. After being washed, one or two of these slides were processed through up-graded ethanol and xylene, and mounted with Eukit for light microscopy. Others were post-fixed in $1 \%$ osmium tetroxide at $4^{\circ} \mathrm{C}$ for $1 \mathrm{hr}$ and dehydrated through up-graded ethanol. After replacement with propylene oxide and Epon-Araldite mixture, gelatin capsules filled with Epon-Araldite mixture were inverted over the tissue sections and the plastics were polymerized on the specimens at $60^{\circ} \mathrm{C}$. The blocks were separated from the slides by heating over a gas burner. Ultrathin sections with or without staining were observed with a JEM $100 \mathrm{~B}$ electron microscope. Control studies were carried out under the following conditions: (a) The peroxidase-labeled antibody was omitted and only peroxidase reaction was performed. (b) The specimens on the slides were pretreated with rabbit antihuman IgA antibody (DAKO PAP, Lot No. 051B) at a
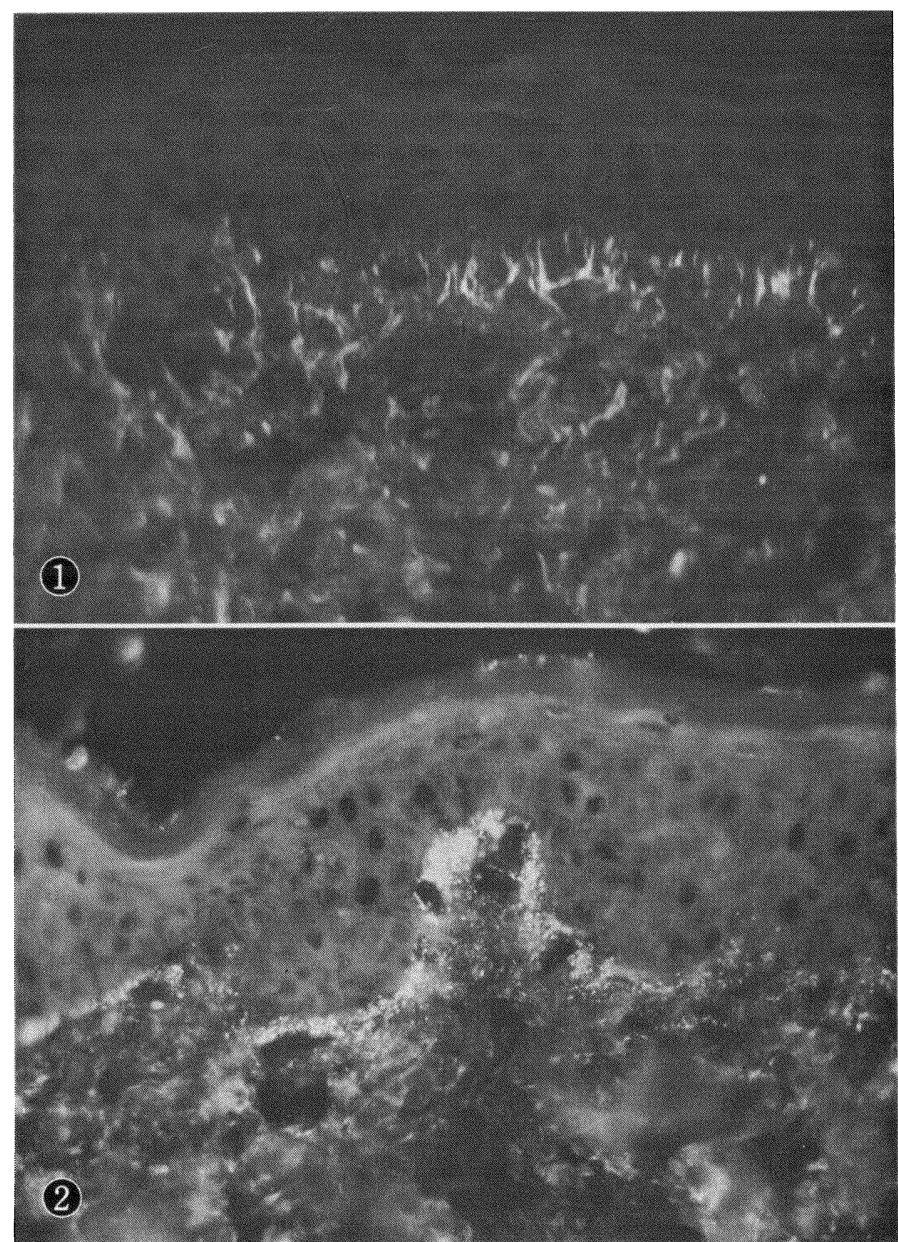

Fig. 1. Fibrillar immunofluorescence pattern of $\operatorname{IgA}$ at the dermal papillae.

Fig. 2. Fine granular immunofluorescence pattern of IgA at the dermal papillae. 
dilution of $1: 5$, then the peroxidase-labeled rabbit anti-human IgA antibody was applied (blocking reaction).

\section{RESUlts}

\section{Histopathology}

The lesional skin showed subepidermal bulla formation with prominent neutrophilic infiltration in all cases. In Cases 1 and 3 , microabscesses were found at the dermal papillae near the bulla.

\section{IF study}

In Cases 1 and 2, direct IF study showed fine fibrillar deposition of IgA at the dermal papillary tips (Fig. 1). In Case 3, however, there were fine granular deposits of $\mathrm{IgA}$ at the dermal papillary tips (Fig. 2). Other immunoglobulins and $\mathrm{C}_{\mathbf{3}}$ were not detected in any cases. The fibrillar or granular pattern of IgA deposition

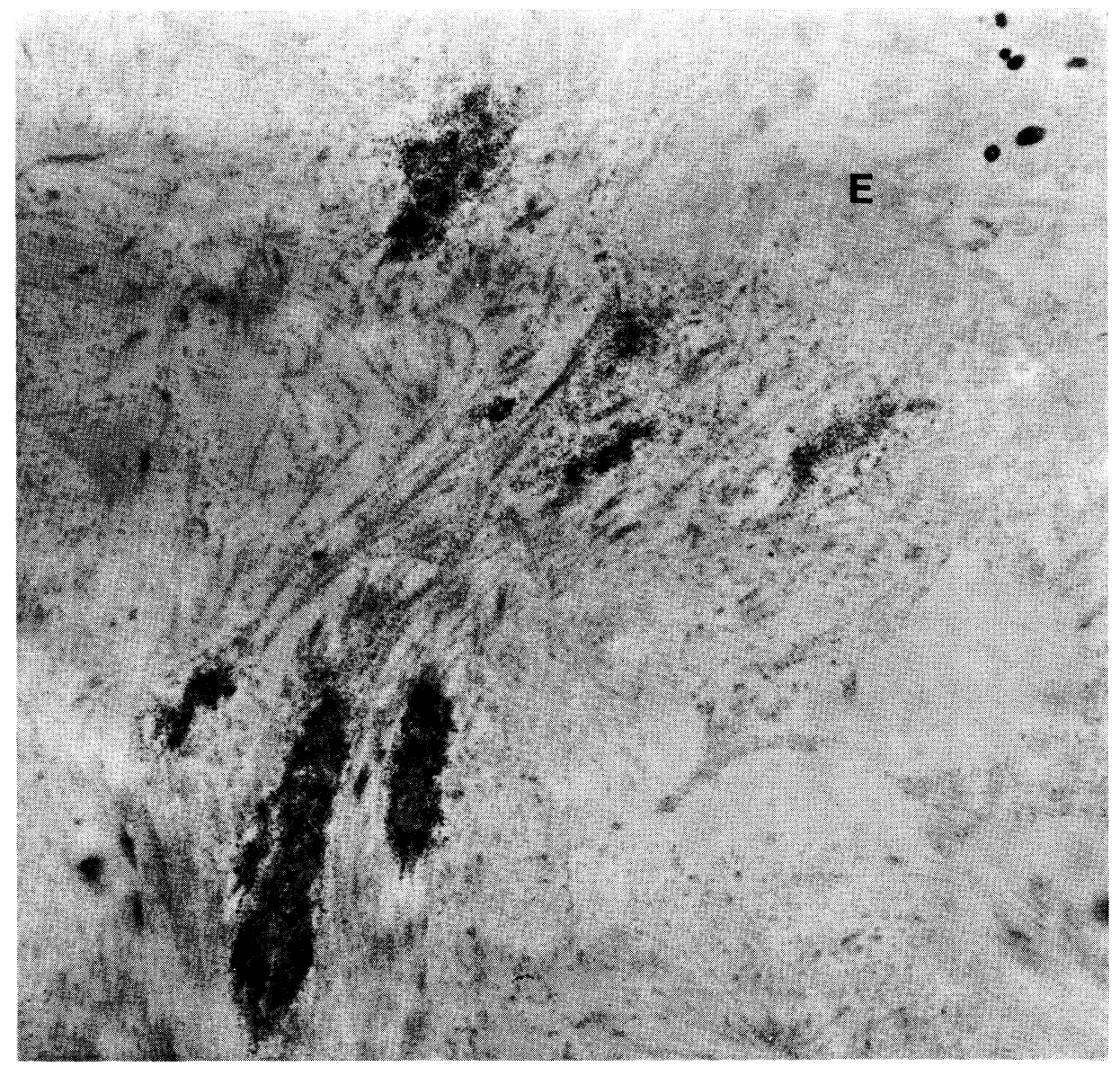

Fig. 3. Focal aggregates of electron-dense reaction products are found in the upper dermis. $\mathrm{E}$, epidermis. $\times 12,000$. 
was constantly seen in each case even though the tissue specimens were cut at various angles. Also, the second biopsies performed in Cases 1 and 3 showed the same results as in the first biopsies of each case. The specific fluorescence was usually more intense in the normal appearing skin than in the lesional skin. No circulating antibodies against the upper dermal components could be detected in any cases by indirect IF study.

\section{IEM study}

In Cases 1 and 2, focal aggregates of electron-dense reaction products were found in the tips of dermal papillae (Fig. 3). Most of them were slender in shape and were occasionally distributed perpendicularly to the epidermis. The reaction products were clearly discernible even after the sections were stained with lead citrate and uranyl acetate (Fig. 4a). At a higher magnification the reaction products were recognized as being very fine granules as shown in Fig. 4b. When

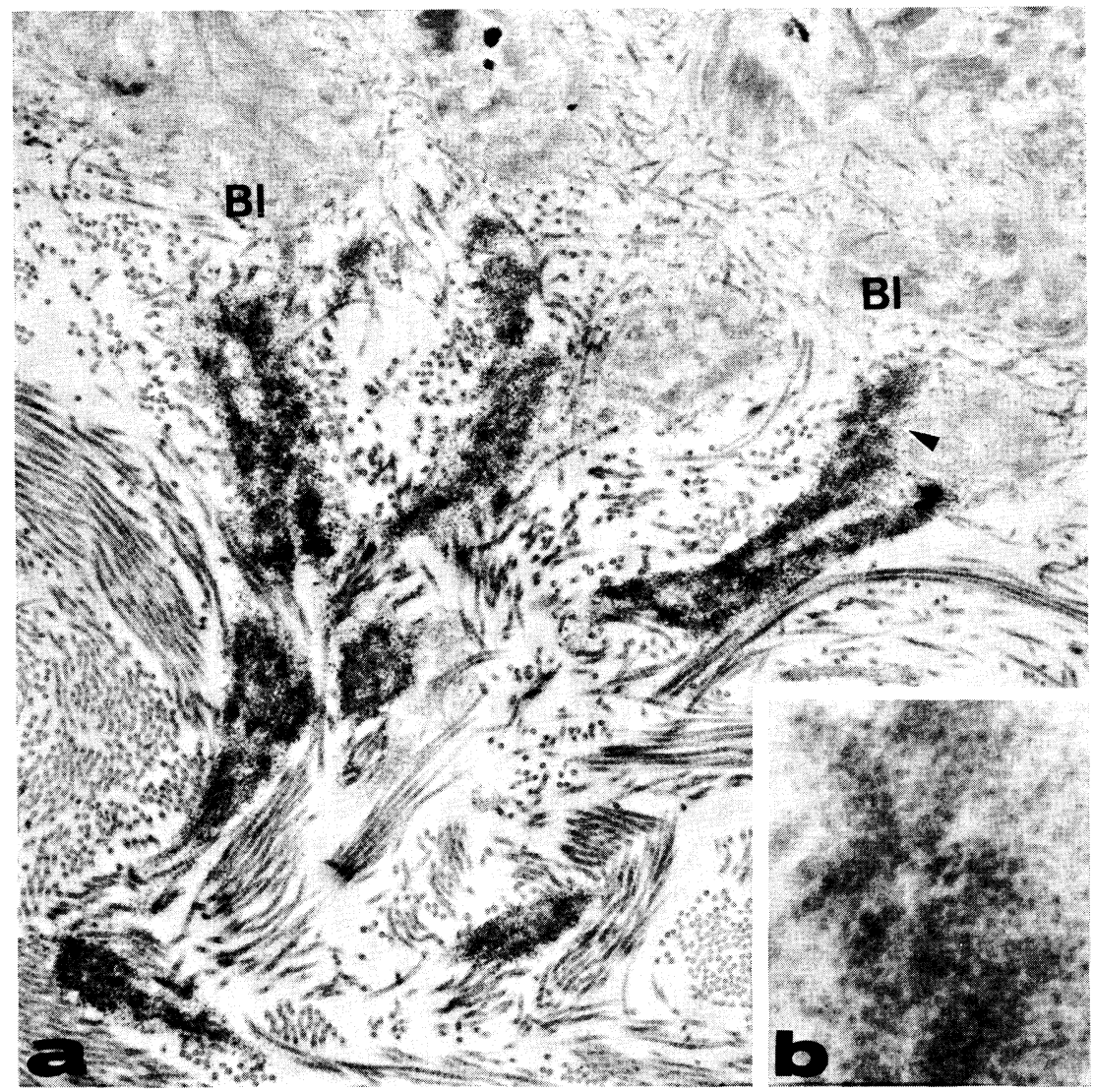

Fig. 4. (a) Even after the sections are stained with lead citrate and uranyl acetate, the reaction products are clearly discernible. They are arranged perpendicularly to the epidermis. Bl, basal lamina. $\times 12,000$. (b) High magnification of the area labeled with an arrow-head in Fig. 4a. The reaction products are recognized as very fine granules. $\times 48,000$. 


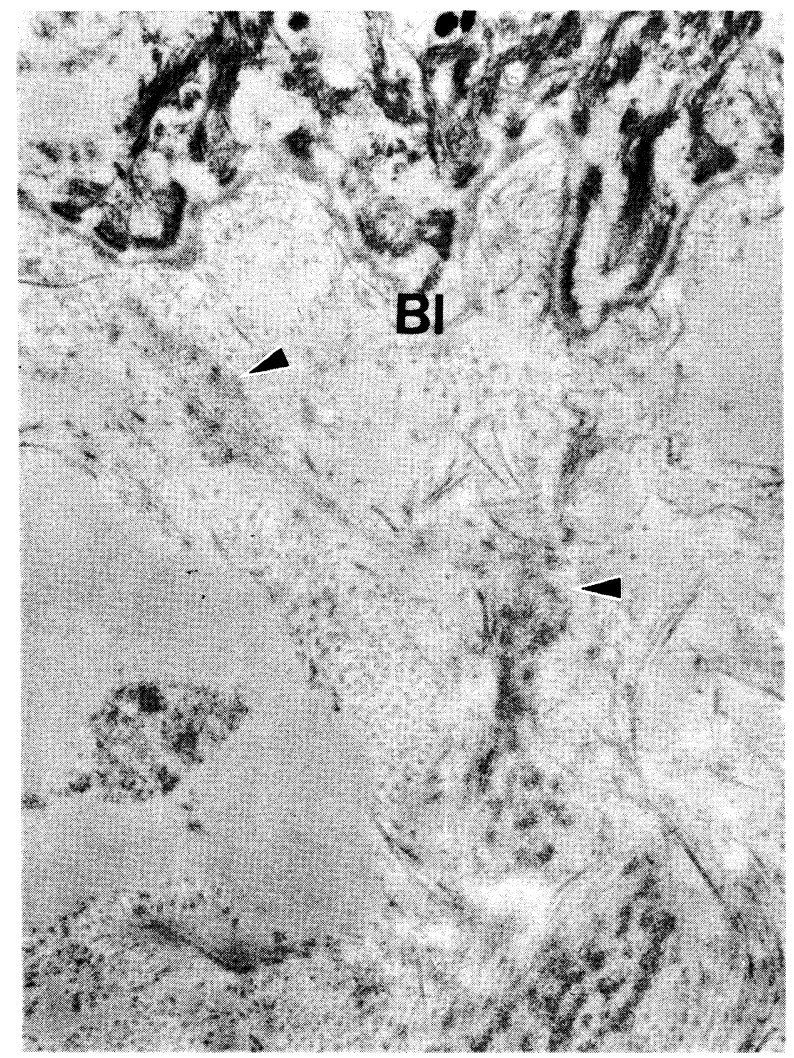

Fig. 5. In the control sections, no reaction products are found on the dermal microfibrillar bundles (arrow-heads). Bl, basal lamina. This is stained with lead citrate and uranyl acetate. $\times 15,000$.

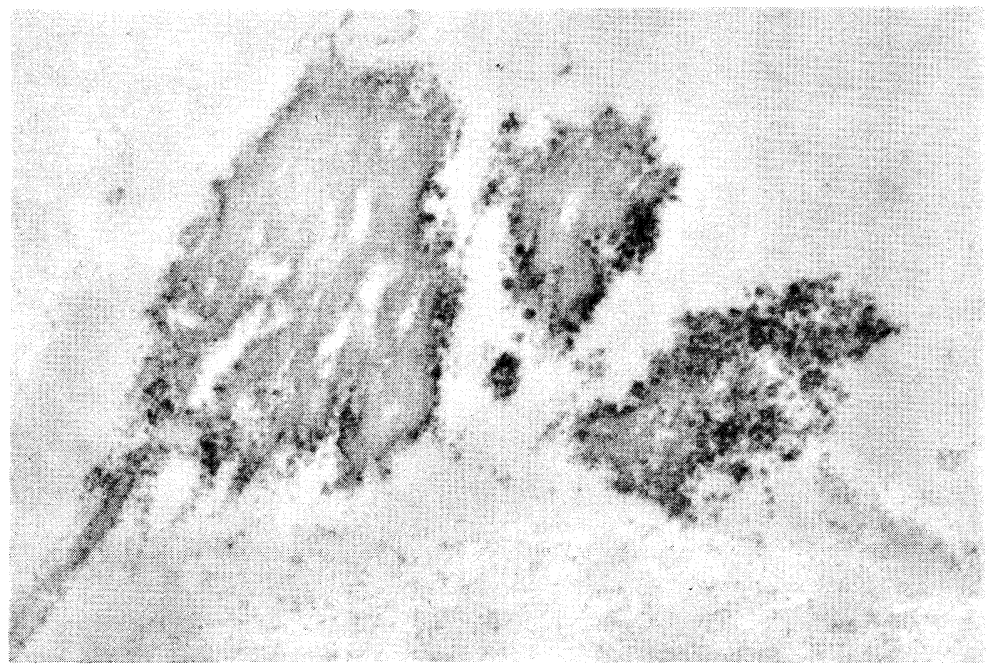

Fig. 6. A few reaction products are found at the periphery of elastic fibers. This section is not stained with lead citrate or uranyl acetate. $\times 30,000$. 


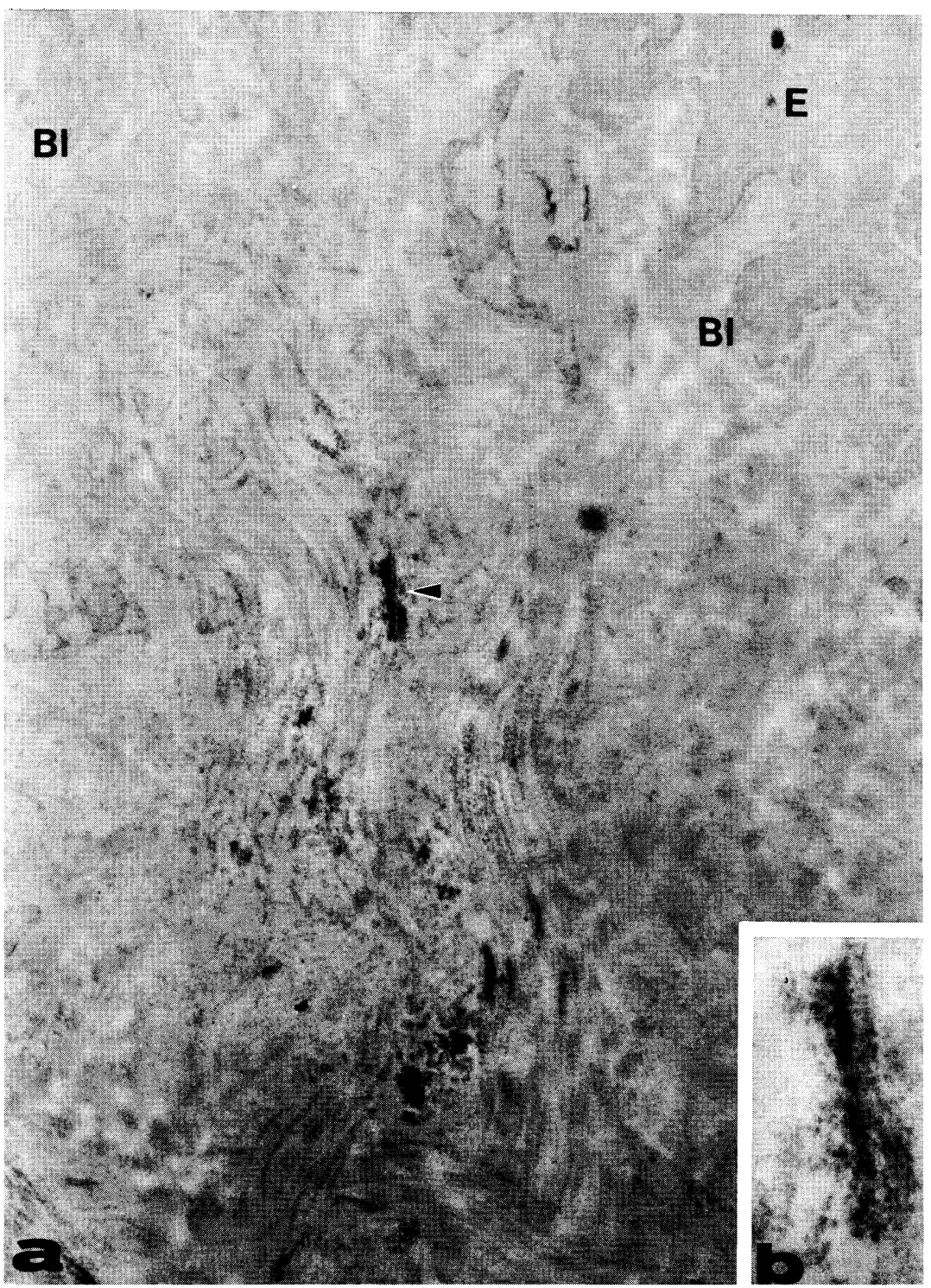

Fig. 7. (a) Smaller aggregates of the reaction products are scattered in the dermal papillae. Bl, basal lamina; E, epidermis. This is not stained with lead citrate or uranyl acetate. $\times 12,000$. (b) High magnification of a part (arrow-head) of Fig. $7 \mathrm{a}$ discloses the close association between the reaction products and a collagen fibril. $\times 48,000$.

compared with the control specimens (Fig. 5), the reaction products appeared to be associated with the microfibrillar bundles of the elastic tissue. In the lower part of the dermal papillae, fewer reaction products were also found at the periphery of elastic fibers (Fig. 6), occasionally in linear distribution, and appeared to be associated with the microfibrillar component of elastic fibers. On the other hand, 


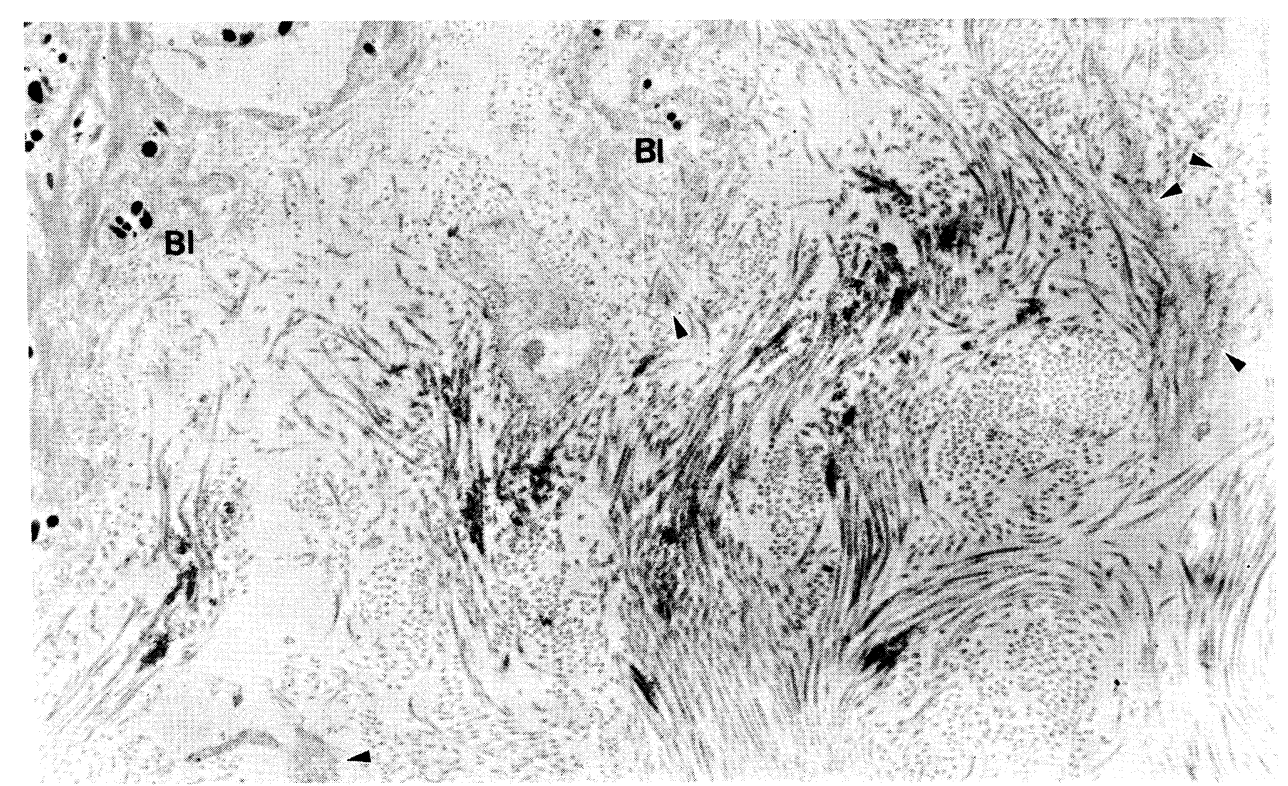

Fig. 8. The association between the reaction products and collagen fibrils are more clearly observed in the stained section. The microfibrillar bundles (arrow-heads) lack the reaction products. $\mathrm{Bl}$, basal lamina. $\times 9,000$.

in Case 3, smaller aggregates of the reaction products were scattered in the dermal papillae (Fig. 7a). At a higher magnification, they were seen to be deposited on and around the collagen fibrils as shown in Fig. $7 \mathrm{~b}$. When the sections were stained with lead citrate and uranyl acetate the association between the reaction products and collagen fibrils became more distinct and the elastic tissues lacked the reaction products (Fig. 8). In the control specimens, such reaction products were not found.

\section{Discussion}

In previous IEM studies of DH cases whose IF patterns of IgA deposition were papillary (granular or fibrillar), it has been reported that the reaction products of $\operatorname{IgA}$ were found to be associated with microfibrillar bundles and with the microfibrillar component of elastic tissue (Stingl et al. 1976; Yaoita and Katz 1976; Schmitt et al. 1977; Yaoita 1978; Pehamberger et al. 1979). In two (Cases 1 and 2) of the present cases, whose IF patterns were fibrillar, the reaction products were obviously associated with those structural components as in previous reports. The distribution pattern of the reaction products, as seen in Figs. 3 and 4, appears to correspond exactly to the perpendicularly arranged brush work of fibers (i.e., dermal microfibrillar bundles) observed on orcein-stained specimens in light microscopy (Pinkus 1970; Briggaman and Wheeler 1975). On the other hand, in Case 3, whose IF pattern was fine granular, the reaction products of IgA were found on and around the collagen fibrils in the dermal papillae and not on elastic 
tissue. Until now, such an ultrastructural localization of IgA has not been reported in DH. It is known, however, that there are variations of the ultrastructural localization of $\mathrm{IgA}$ in the DH cases with the linear or continuous IF pattern, that is, at the lamina lucida or below the basal lamina (Yaoita and Katz 1976; Dabrowski et al. 1979). So it is possible that there are also some variations of IgA localization in the $\mathrm{DH}$ cases with papillary IF pattern. In general, it is thought that the difference in IF pattern, fibrillar or granular, is due to whether the tissue specimens are sectioned longitudinally or transversely (Seah and Fry 1975). Igarashi et al. (1982), however, reported that the appearance of the fibrillar pattern of $\operatorname{IgA}$ deposition did not alter even though the tissue specimens were cut at various angles. We also obtained the same results in this respect. So there may arise another point of view that the granular pattern of $\operatorname{IgA}$ deposition may be essentially different from the fibrillar pattern as to the ultrastructural localization of IgA. But further IEM studies of many DH cases will be necessary to support this possibility, and the reason for such variations of $\operatorname{IgA}$ localization in $\mathrm{DH}$ skin also must be explained.

In recent reviews of Japanese cases of DH (Igarashi et al. 1982; Tanita et al. 1982), it was reported that no Japanese cases of DH had HLA-B8; this antigen has frequently been detected in Western cases of DH with papillary (fibrillar or granular) IgA deposition (Fry 1979). Likewise, none of the present cases had HLA-B8. It may be also possible that Japanese cases of DH have somewhat different nature from Western cases.

\section{References}

1) Briggaman, R.A. \& Wheeler, C.E., Jr. (1975) The epidermal-dermal junction. $J$. invest. Derm., 65, 71-84.

2) Dabrowski, J., Chorzelski, T., Jablonska, S., Krainska, T. \& Jarzabek-Chorzelska, M. (1979) Immunoelectron microscopic studies in IgA linear dermatosis. Arch. derm. Res., 265, 289-298.

3) Fry, L. (1979) Dermatitis herpetiformis: Basic findings. In: Immunopathology of the Skin. 2nd ed., edited by E.H. Beutner, T.P. Chorzelski \& S.F. Bean, Wiley Medical Publication, New York, pp. 283-301.

4) Igarashi, R., Sudo, S., Morohashi, M. \& Oka, K. (1982) Dermatitis herpetiformis Duhring-Relationship between fibrillar and granular pattern. Jap .J. clin. Derm., 36, 333-338. (Japanese)

5) Graham, R.C. \& Karnovsky, M.J. (1966) The early stages of absorption of injected horse-radish peroxidase in the proximal tubules of mouse kidney: Ultrastructural cytochemistry by a new technique. J. Histochem. Cytochem., 14, 291-302.

6) Masu, S., Yokota, M., Tanita, Y., Demitzu, T., Miura, T. \& Seiji, M. (1982) Statistical observation of bullous dermatoses; Pemphigus, bullous pemphigoid and dermatitis herpetiformis Duhring. Jap. J. clin. Derm., in press. (Japanese)

7) McLean, I.W. \& Nakane, P.K. (1974) Periodate-lysine-paraformaldehyde fixative. A new fixative for immunoelectron microscopy. J. Histochem. Cytochem., 22, 10771083.

8) Pehamberger, H., Konrad, K. \& Holubar, K. (1979) Juvenile dermatitis herpetiformis: An immunoelectron microscopic study. Brit. J. Derm., 101, 271-277.

9) Pinkus, H. (1970) The direction of growth of human epidermis. Brit. J. Derm., 83, $556-564$. 
10) Seah, P.P. \& Fry, L. (1975) Immunoglobulins in the skin in dermatitis herpetiformis and their relevance in diagnosis. Brit. J. Derm., 92, 157-166.

11) Schmitt, D., Germain, D. \& Thivolet, J. (1977) A simple technique for immunoelectronmicroscopic localization of Ig on tissue sections with special reference to cutaneous pathology. Arch. derm. Res., 259, 235-245.

12) Stingl, G., Honigsmann, H., Holubar, K. \& Wolff, K. (1976) Ultrastructural localization of immunoglobulins in skin of patients with dermatitis herpetiformis. J. invest. Derm., 67, 507-512.

13) Tanita, Y., Masu, S.,Tomita, Y.\& Miura, T. (1982) Two cases of dermatitis herpetiformis Duhring. Rinsho Derm. (Tokyo), in press. (Japanese)

14) Yaoita, H. (1978) Identification of IgA binding structures in skin of patients with dermatitis herpetiformis. J. invest. Derm., 71, 213-216.

15) Yaoita, H. \& Katz, S.I. (1976) Immunoelectronmicroscopic localization of IgA in skin of patients with dermatitis herpetiformis. J. invest. Derm., 67, 502-506. 